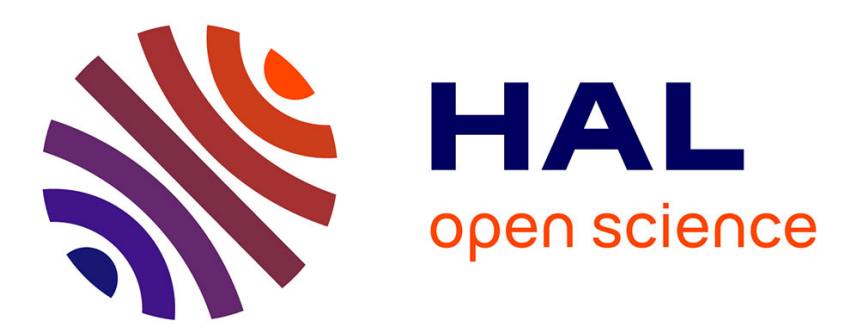

\title{
Thermo-Mechanical Investigations of a Tribological Interface
}

\author{
Jérôme Rivière, Mathieu Renouf, Yves Berthier
}

\section{To cite this version:}

Jérôme Rivière, Mathieu Renouf, Yves Berthier. Thermo-Mechanical Investigations of a Tribological Interface. Tribology Letters, 2015, 58, pp.48-59. 10.1007/s11249-015-0523-4 . hal-01233087

\section{HAL Id: hal-01233087 \\ https://hal.science/hal-01233087}

Submitted on 24 Nov 2015

HAL is a multi-disciplinary open access archive for the deposit and dissemination of scientific research documents, whether they are published or not. The documents may come from teaching and research institutions in France or abroad, or from public or private research centers.
L'archive ouverte pluridisciplinaire HAL, est destinée au dépôt et à la diffusion de documents scientifiques de niveau recherche, publiés ou non, émanant des établissements d'enseignement et de recherche français ou étrangers, des laboratoires publics ou privés. 


\title{
Thermo-Mechanical Investigations of a Tribological Interface
}

\author{
Jérôme Rivière $^{1} \cdot$ Mathieu Renouf $^{2} \cdot$ Yves Berthier $^{1}$
}

\begin{abstract}
Numerical methods are essential to understand tribological behaviors since it is difficult to measure directly a closed contact or write representative analytical equations. In this paper, a focus on the complexity of a contact is done with the modeling of thermo-mechanical phenomena in connection with tribological triplet (mechanism, first bodies, third body). Discrete element method is chosen to have a dynamic view of a contact and is interesting to represent both damage of first bodies and cohesion of third ones. Thermo-mechanical models are described for first and third-bodies and are adjusted as a function of continuity of the body. Results regarding damage, rheology and thermal effects are studied as a consequence of cohesion of third body and applied energy by the mechanism (pressure, velocity). Because mechanical and thermal behaviors have a narrow but unclear relationship, a balance between local energy (cohesion of third body) and global energy (applied forces by mechanism) is recommended.
\end{abstract}

Keywords Discrete element method (DEM) - Thermal contact conductance (TCC) · Damage · Third body · Continuous equivalent media

Jérôme Rivière

jerome.riviere@insa-lyon.fr

1 LaMCoS, INSA Lyon, UMR 5259, Université de Lyon, CNRS, 69621 Villeurbanne Cedex, France

2 LMGC, UMR 5508, Université Montpellier 2, CNRS, 34095 Montpellier Cedex, France

\section{Introduction}

The relative motion of two bodies in contact is the seat of several dissipative phenomena $[1,2]$. In particular, one part of the necessary mechanical energy to rub bodies against each other is converted into thermal energy (from 80 to $95 \%$ according to the literature $[3,4])$. Under dry contact conditions, this conversion can lead to hot spot localization [5] and thermoelastic instabilities [6] or to the alteration of mechanical, thermal and chemical properties [7].

The literature proposes a large variety of analytical models trying to represent the contact complexity [8,9], based on roughness assumptions, plastic deformation and wear flows [10]. It appears that there are only few models accounting for the dynamic evolution of the contact, leading to the creation of an interfacial layer composed for the most part of the transformation and the degradation of debris particles issued from the bodies in contact. This layer, usually called the third body in reference of the two bodies in contact [11], is well known to its mechanical roles (velocity accommodation, load transmission,...) but less for its thermal ones. The main reason is the confined feature of the contact, which makes difficult its local dynamic instrumentation. Only external measures can be performed, giving global information that not always corresponds to the reality of the contact $[2,12,13]$.

Before Godet's concept, Ryhming [14] was the first to underline the fact that the discontinuous contact interface (not yet called "third body") is at the origin of the thermal energy dissipation in the contact during chip removal machining. The main consequence of his work was to find possible different surface temperatures for the two "contacting" bodies. However, this approach does not take into account the local rheology of the third body and its participation in the velocity accommodation during the sliding 
process. The heat generation is still considered constant through the third-body thickness and depends on the global friction coefficient, experimentally determined, thanks to the normal pressure and the shear velocity.

With the development of discrete element methods (DEM) [15] and their extensions to thermo-mechanical behavior of contact interface [16], it is possible to analyze numerically the life of a contact. Several results have been observed [16] as, for example, the localization of the maximal temperature within the thickness of the third body as a function of its internal cohesion. Nevertheless, the approach stays at the scale of the third body, and the influence of first bodies is related to some specific thermal boundary conditions.

This paper proposes a new approach to model the thermo-mechanical behavior of a tribological interface based on an equivalence between continuous and discontinuous thermal models. It accounts for the continuous feature of the bodies in contact and their damage until the creation of debris particles. This model uses the conceptual approach of the tribological triplet $[11,17]$ and the tribological circuit model [18], which are tools for understanding and interpreting wear processes. They provide descriptive schemes which can lead to comprehensive models. This new approach presented in the paper relies on a DEM used as a meshless approach to investigate contact problems from a mechanical point of view only [19]. The method is extended to account for thermal feature such as heat generation and heat conduction through a continuous, a damaged or a discontinuous equivalent media. Static validations are performed and compared to results obtained with a finite-element method. Discussions and energy consideration are performed around an academic tribological case (elementary cell under normal pressure and shearing velocity). A focus is performed on the competition between the global applied energy and the local one through interaction forces.

\section{Numerical Mechanical Framework}

The method used to simulate evolution of a divided media is the non-smooth contact dynamics (NSCD) approach, developed by Moreau [20] and Jean [15]. This approach has been widely used for tribological application dealing with mechanical [21], thermal [22] or more complex behaviors [22]. The approach differs from other approaches offered in the literature by its both implicit time integration and contact resolution, guaranteeing a converging stability and avoiding numerical tuning.

Recently, the approach has been extended [23] and used as a meshless approach to model equivalent continuous media [19]. Cohesive zone models (CZMs) [24] have been used to confer with the whole packing a continuous behavior. This model includes an internal variable, denoted $\beta[25](\beta \in[0,1])$, which represents the adhesion intensity of the interaction. Physically, it accounts for proportion of microstructure defects like fractures, cracks or porosity. When $\beta$ equals to 1 , the interaction is not damaged, while it is broken when $\beta$ equals to 0 .

Thus, to model degradation of a first body and creation of a third-body layer, two interaction laws are defined to represent the different states.

Usually, the CZM used to describe interaction between first-body particles is based on five parameters: $C_{\mathrm{n}}$ and $C_{\mathrm{t}}$, the normal and tangential stiffness, respectively; $w$, the decohesion energy; $b$, the viscosity associated with evolution of inter-particle adhesion and $\mu$ the local friction coefficient. For purposes of the present work, friction is equal to zero $(\mu=0)$, like viscosity $(b=0)$. Such parameters can be related to the packing rigidity. Indeed, this last one is already guaranteed by both the high volume fraction of the packing and the high normal stiffness. Thus in the present case, CZM friction and viscosity are secondary.

Initial interactions are perfectly intact $(\beta=1)$ and normal and tangential stiffness are considered equal. This assumptions means that the local model is defined by two parameters, i.e., $C_{\mathrm{n}}$ and $w$ :

$\sigma=C_{\mathrm{n}}(\beta) \epsilon$,

where $\sigma$ and $\epsilon$ are, respectively, the local stress and strain. The elastic stress threshold $\sigma_{c}$ is equal to $\sqrt{w C_{\mathrm{n}}}$. If the value of $\sigma$ is less than this threshold, the damage parameter does not evolve. On the over case, $\beta$ evolves, modifying both normal and tangential stiffnesses.

For debris particles, interactions are governed by a nonsmooth contact cohesive law used to reproduce reversible cohesion due to physicochemical effects [22]. Let $d_{w}$ be the attraction distance between two particles, $g$, the gap between particles and $r_{\mathrm{n}}$ the normal contact force; one obtains a linear complementarity problem such that:

$g \geq 0, \quad r_{\mathrm{n}}+\gamma=0, \quad g \cdot\left(r_{\mathrm{n}}+\gamma\right)=0$

where $\gamma$ denotes the cohesion intensity. The cohesive condition is obtained when $\gamma<r_{\mathrm{n}}<0$ and $g \leq d_{w}$. The stick condition is reached when $r_{\mathrm{n}} \geq 0$ and $g=0$. The decohesion condition is applied when $r_{\mathrm{n}}<\gamma$ or $g>d_{w}$.

Note that even if no tangential component is defined concerning local contact forces, it will be possible to measure a macroscopic friction coefficient using rough boundaries. The influence of the local friction on the macroscopic friction is secondary in regard to cohesion and roughness used in the model [26]. 


\section{Heat Generation and Conduction in Discrete Media}

Thanks to previous work, it is possible to model heat generation and transfer in discrete media [27, 28]. According to the chosen model, the temperature of particles in a discrete medium can be tracked considering conductive, radiative or convective effects leading to the thermal evolution Eq. (3):

$\rho_{i} c_{i} V_{i} \frac{\mathrm{d} T_{i}}{\mathrm{~d} t}=Q_{i}^{\text {gen }}+Q_{i}^{\text {cond }}+Q_{i}^{\text {conv }}+Q_{i}^{\text {rad }}$

where $\rho_{i}$ is the mass density, $c_{i}$, the specific heat and $V_{i}$, the volume of particle $i$. $Q_{i}^{\text {cond }}$ corresponds to the local conductive heat flux received by the element $i j$ subjected to multiple static interactions. $Q_{i}^{\text {gen }}$ is the local generative heat flux produced when two particles enter in contact dynamically, $Q_{i}^{\text {conv }}$ is the local convective heat flux with a fluid environment, and $Q_{i}^{\text {rad }}$ is the local radiative heat flux exchanged. In the case of a tribological interface where the set of particles represents an equivalent volume, $Q_{i}^{\text {conv }}$ and $Q_{i}^{\text {rad }}$ are not of the first order [16]. They are considered as null in the proposed model.

\subsection{Heat Generation}

When two particles come in contact, the variation of their kinetic energy can be source of heat generation. This energy, denoted $\mathcal{E}_{i j}$, is written as:

$\mathcal{E}_{i j}=\mathbf{r}_{\alpha} \mathbf{v}_{\alpha}$

where $\mathbf{r}_{\alpha}$ is the local contact force and $\mathbf{v}_{\alpha}$ the contact relative velocity associated with contact $\alpha$. Then, this energy is assumed to be split into two equal parts. Thus according to such hypotheses, for the contact $\alpha$ involving particles $i$ and $j$, the local generated heat flux $Q_{i j}^{\text {gen }}$ acting on a particle is written as:

$Q_{i j}^{\text {gen }}=\frac{\psi}{2} \mathcal{E}_{i j}$.

The parameter $\psi$ is introduced to consider, eventually, only a part of such an energy [8]. Nevertheless, as proposed in [22], other forms of energy transformation, such as convertion into vibration, phase transformation or volumetric heat by plastic strain [8], are not considered, and all the dissipated mechanical energy is converted into thermal energy (i.e., $\psi=1$ ). Note that even if a value of $\psi \neq 1$ changes the amount of generated heat, it will be changed in the same way for all simulations and, consequently, does not affect quantitative comparisons.

\subsection{Heat Transfer}

In the literature, the works of Yovanovich act as a reference to model heat transfer between rigid bodies [29, 30]. Vargas and McCarthy [27] were the first to use such model in large discrete element assemblies. Considering the contact $\alpha$ involving particles $i$ and $j$, the local conductive heat flux can be expressed as:

$$
Q_{i j}^{\text {cond }}=H\left(T_{i}-T_{j}\right),
$$

where $T_{i}$ and $T_{j}$ are, respectively, temperature of discrete elements $i$ and $j$ and $H$, the thermal contact conductance (TCC), which contains the thermal behavior related to the microstructural evolution of contact. Several authors work on an accurate representation of $H$ to obtain a better estimation of contact temperatures [29-31]. However, they all have a static view of the contact concerning asperities for a two bodies contact model.

In discrete assemblies, $H$, denoted $H^{d}$, derives from Hertz's elastic contact theory [32]:

$H^{d}=2 k_{\mathrm{th}} a$.

It is the product of the thermal conductivity $k_{\mathrm{th}}$ and the contact area radius $a$. In a two-dimensional problem, a contact between two disks must be seen as a contact between two cylinders of unit length $(L=1)$. In this case, from Eq. (7), $a$ represents the half-width of the rectangle contact area:

$a=\sqrt{\frac{4\left(r_{n}+\gamma\right) r^{*}}{\pi L E^{*}}}$,

where $r^{*}$ and $E^{*}$ are, respectively, the effective radius and Young modulus. Note that Eq. (8) is extended to cohesive contact by adding up the contact force with the absolute value of the cohesive force $\gamma$ [22].

\section{Heat Transfer in Continuous Equivalent Media}

\subsection{Formulation}

When DEMs are used to represent an equivalent continuous medium, Eq. (8) cannot be used directly without increasing its thermal resistance. The TCC should be adapted to obtain an equivalent behavior from a thermal point of view.

This adaptation relies on an analogy between the discretization of a medium using both a disk stacking in a regular square network and a finite difference method (FDM) (cf Fig. 1). If the nodes of FDM are associated with 


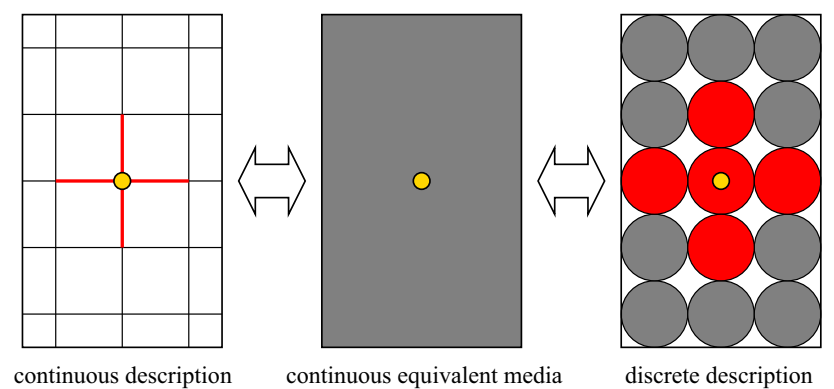

Fig. 1 Thermal analogy between continuous and discrete models

a disk, the temperature of a point of medium can be estimated from a continuous or a discrete way.

To illustrate this approach, let us consider a solid plate of length $L$ and width $W$, divided in regular cells of dimensions $2 r \times 2 r$ where $r$ is the radius of disks using to discretize the region.

According to Eq. (3), reduced to conduction terms only and discretized by an Euler scheme, the temperature $T$ of the element $i j$ is equal to:

$$
T_{i j}^{+}=T_{i j}^{-}+\frac{\mathrm{d} t}{A}\left[Q_{i j,(i-1) j}^{-}+Q_{i j,(i+1) j}^{-}+Q_{i j, i(j-1)}^{-}+Q_{i j, i(j+1)}^{-}\right] .
$$

where $A$ is equal to $\rho c V$ and considered constant for all elements. Exponents + and - refer to variables computed at end and beginning of time step (of length $\mathrm{d} t$ ).

Each conductive heat flux $Q_{\ldots \ldots}^{-}$in Eq. (9) can be replaced by its value given by Eq. (6). Assuming that $H$, denoted $H^{\mathrm{c}}$, is constant for each couple of elements, one obtains:

$$
\begin{aligned}
T_{i j}^{+}= & T_{i j}^{-}+\frac{\mathrm{d} t}{A} H^{\mathrm{c}}\left[\left(T_{(i-1) j}^{-}-T_{i j}^{-}\right)+\left(T_{(i+1) j}^{-}-T_{i j}^{-}\right)\right. \\
& \left.+\left(T_{i(j-1)}^{-}-T_{i j}^{-}\right)+\left(T_{i(j+1)}^{-}-T_{i j}^{-}\right)\right],
\end{aligned}
$$

equivalent to:

$$
\begin{aligned}
T_{i j}^{+}= & \left(1-\frac{4 \mathrm{~d} t}{A} H^{\mathrm{c}}\right) T_{i j}^{-} \\
& +\frac{\mathrm{d} t}{A} H^{\mathrm{c}}\left(T_{(i-1) j}^{-}+T_{(i+1) j}^{-}+T_{i(j-1)}^{-}+T_{i(j+1)}^{-}\right),
\end{aligned}
$$

which corresponds to the discrete formulation of the temperature evolution of element $i$.

This evolution can now be seen from a continuous point of view using the classical heat equation:

$\rho c \frac{\mathrm{d} T}{\mathrm{~d} t}=k_{\mathrm{th}}\left(\frac{\partial^{2} T}{\partial x^{2}}+\frac{\partial^{2} T}{\partial y^{2}}\right)$.

If same spatial discretization is used in the $X$ and $Y$ directions $(\mathrm{d} x=\mathrm{d} y)$, the approximation of Eq. (12) by FDM is equal to: (a)

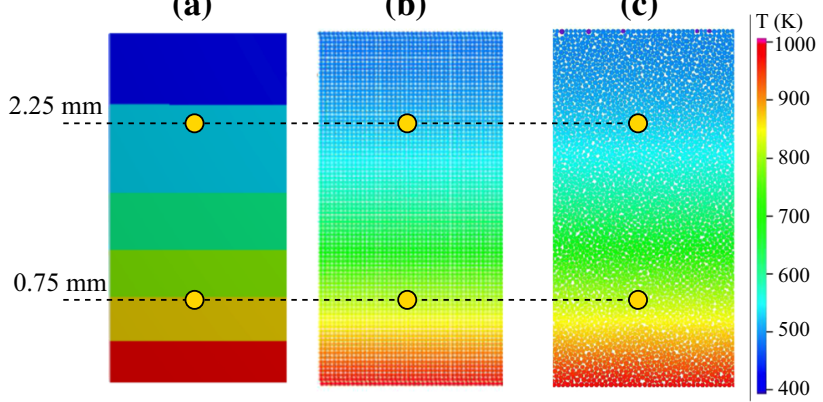

Fig. 2 Thermal field for $t=500 \mathrm{~ms}$ for each discretization: FEM (a), square (b) and random (c)

$$
\begin{aligned}
& \frac{\rho c}{\mathrm{~d} t}\left(T_{i j}^{+}-T_{i j}^{-}\right) \\
& =\frac{k_{\mathrm{th}}}{\mathrm{d} x^{2}}\left(T_{(i-1) j}^{-}+T_{(i+1) j}^{-}+T_{i(j-1)}^{-}+T_{i(j+1)}^{-}-4 T_{i j}^{-}\right) .
\end{aligned}
$$

Using a spatial discretization equal to $2 r$, temperature evolution of node $i j$ is given by:

$$
\begin{aligned}
T_{i j}^{+}= & {\left[1-\frac{\mathrm{d} t k_{\mathrm{th}}}{\rho c r^{2}}\right] T_{i j}^{-} } \\
& +\frac{\mathrm{d} t k_{\mathrm{th}}}{4 \rho c r^{2}}\left[T_{(i-1) j}^{-}+T_{(i+1) j}^{-}+T_{i(j-1)}^{-}+T_{i(j+1)}^{-}\right] .
\end{aligned}
$$

Finally, the expression of $H^{\mathrm{c}}$ comes from the identification of Eqs. (11) and (14). In a two-dimensional problem, as a disk is considered as a cylinder of unit length, the thermal capacity $A$ is equal to $\rho c \pi r^{2}$. Consequently, one obtains for the TCC the following equation:

$H^{\mathrm{c}}=\frac{\pi k_{\mathrm{th}}}{4}$.

Finally, one can note that Eq. (15) represents the splitting of heat flux in four parts, corresponding to the four neighbors of a particle in a square lattice. To transpose this equation to any packing of disks, the number of contacts of each particle, denoted $v$ and called commonly the "coordinance," needs to be taken into account. Consequently, Eq. (15) evolves into the following equation:

$H^{\mathrm{c}}=\frac{\pi k_{\mathrm{th}}}{v}$.

It is interesting to highlight that $H^{\mathrm{c}}$ depends only on one microstructural parameter $(v)$ and not on numerical ones [33].

\subsection{Numerical Comparisons}

In order to confirm the result given by Eq. (16), evolution of temperature in a solid plate submitted to an external thermal source is simulated. The solid plate is discretized 
by square and random stacks composed of 6241 rigid disks. Results are compared to finite-element simulation where the size of mesh cells is equal to the diameter of one particle (i.e., $0.038 \mathrm{~mm}$ ). Initial temperature $T_{0}$ is $300 \mathrm{~K}$, and a heat source is applied on the lower boundary $\left(T_{s}=1000 \mathrm{~K}\right)$. Adiabatic boundary conditions are used for other boundaries.

Figure 2 exposes the final thermal field for square and random stacks by comparing with a FEM simulation run with ANSYS@.

From a macroscopic and a qualitative point of view, thermal fields resulting from different models seem to be equivalent. To complete previous snapshots with quantitative results, temperature is measured at two points of the structure, located at 0.75 and $2.25 \mathrm{~mm}$ from the heat source (cf Fig. 3).

As was supposed, there is a really good fit between the different curves, independently of the chosen discretization. Consequently, the introduction of the coordinance indicated in Eq. (16) is a good and simple way to be independent of the discretization induced by the packing of disks and to build an equivalent continuous media with appropriate thermal properties.

\subsection{Heat Transfer in Damaged Media}

In previous section, transfer models have been presented for continuous equivalent media as well as for discontinuous one. During tribological solicitations, a third-body layer is created, resulting from damage of first body. Consequently, it is natural to want to transit also for both states from a thermal point of view.

To perform such transition and to propose a model accounting for damage, value of TCC is discussed according to the damage variable $\beta_{\alpha}$ related to CZM and contact conditions. Thus, the TCC of a damage media, denoted $H^{e}$, is governed by the following conditions:
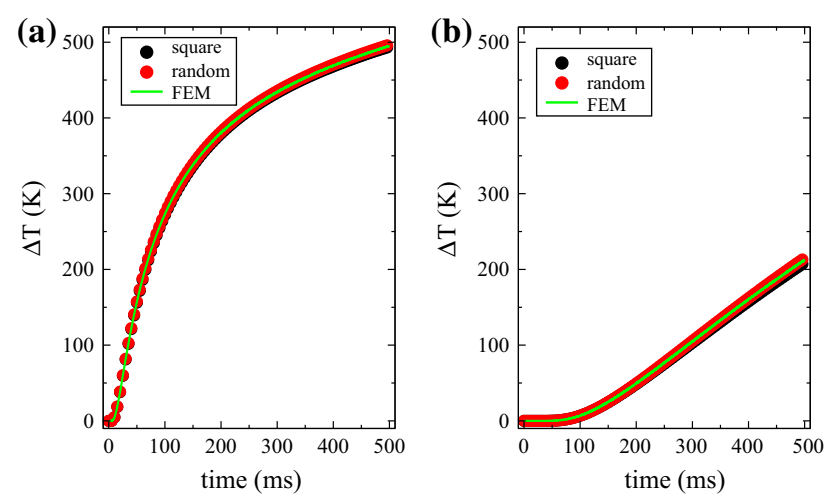

Fig. 3 Temperature evolution at a $0.75 \mathrm{~mm}$ and b $2.25 \mathrm{~mm}$ from heat source with $r=0.019 \mathrm{~mm}$

$$
\begin{array}{ccc}
\text { if } r_{n}<0 & \text { then } & H^{e}=\beta_{\alpha} H^{\mathrm{c}} \\
& \text { else } & H^{e}=\beta_{\alpha} H^{\mathrm{c}}+\left(1-\beta_{\alpha}\right) H^{d} .
\end{array}
$$

Two cases are distinguished in System (17). When the interaction force between two particles is negative (tension condition), continuity of the contact $\alpha$ is only related to the $\beta_{\alpha}$ variable. Heat transfer occurs only through this undamaged part of the contact leading to the first equation in System (17). When interaction force is positive (compression condition), there is a combination between the continuous part of the interface and the contact condition acting on the other part, leading to the ponderation in the second equation in System (17).

\subsection{Thermal Boundary Conditions}

A system without boundary conditions or with uncontrolled boundary conditions could have a major impact on the physical results, especially on the study of rheology of a tribological interface [34]. In such systems, the use of adiabatic environment increases temperature within the interface and exacerbates thermal phenomena. It is then necessary to use methods allowing heat evacuation as proposed in [16]. For the purpose of the present work, the model proposed in [22] is used (cf Fig. 4). It relies on the use of a two-dimensional continuous model to keep a heterogeneous evolution around the boundaries and to find an average behavior at a certain distance from the sample based on Eq. (12), discretized using FDM.

At each time step of the simulation, the considered discrete boundary acts as an interface between the sample and the continuous domain. It serves as an initial condition for lower nodes to solve Eq. (12). Once the equation is solved, it is possible to determine the amount of heat which is spread in the volume by the boundary particles and thus update their temperature.

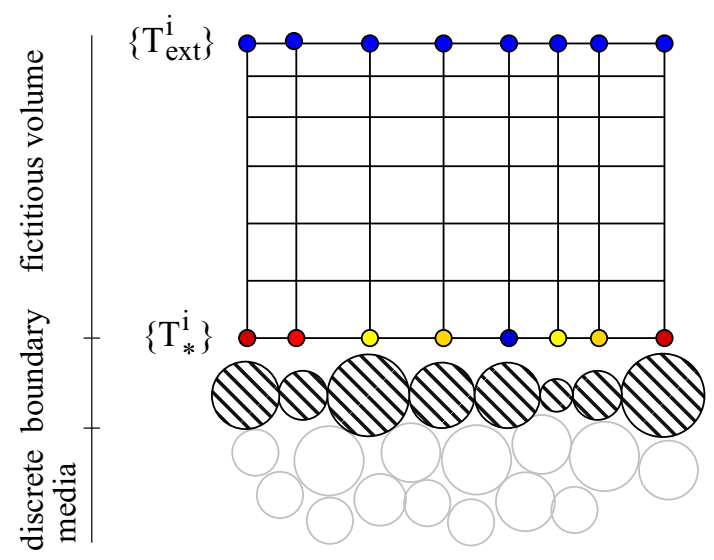

Fig. 4 Two-dimensional thermal boundary conditions 


\section{Tribological Solicitations}

\subsection{Model Description}

The formalism presented in previous sections is applied to a homogeneous sample submitted to global tribological conditions. According to different sets of boundary conditions, the tribological behavior of the sample is investigated in terms of mechanical properties (damage, macroscopic friction) as well as thermal ones (maximal temperature). Figure 5 presents the elementary volume used in the simulations.

Sample length and thickness are both equal to $3 \mathrm{~mm}$. The sample is composed of 6360 rigid disks whose average radius is equal to $19 \mu \mathrm{m}$, connected by CZM (law parameters are summarized in Table 1). Upper and lower boundaries are represented by two rough walls. The upper boundary, submitted to a normal pressure $P$, is connected to the packing with CZM. The lower boundary moves with a content velocity $V$ and interacts with the packing according to the unilateral cohesive law. Periodical conditions are imposed in the shear direction (lateral boundaries): Particles on the right of the sample are in contact with particles on the left (wear is neglected).

The thermal boundary conditions presented in Sect. 4.4 are applied on the upper and lower boundaries to allow a heat transfer out the contact area (cf Fig. 5b). The height $H$ of the two fictitious domains is larger than the height $h$ of the sample (i.e., $H / h=10$ ). For each boundary, nodes on the left of the domain have the same temperature as the nodes on the right (i.e., $\forall y \in[0, H], T(0, y)=T(L, y)$ ). Periodical conditions imposed in the shear direction allow heat transfer between particles on the left in contact with particles on the right of the sample. (a)

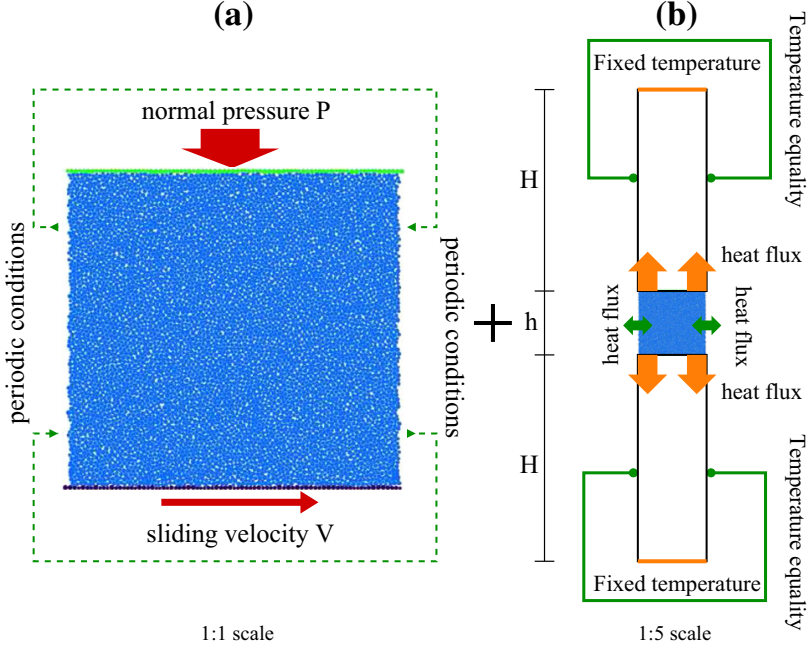

Fig. 5 Mechanical (a) and thermal (b) boundary conditions applied on a homogeneous sample under tribological conditions
Table 1 Sample parameters

\begin{tabular}{ll}
\hline Number of particles & 6360 \\
Length $(\mathrm{mm})$ & 3 \\
Thickness $(\mathrm{mm})$ & 3 \\
Average radius (mm) & 0.019 \\
Time step (ms) & $10^{-5}$ \\
Simulation time (ms) & 10 \\
$\rho\left(\mathrm{g} \mathrm{mm}^{-3}\right)$ & $1.75 \times 10^{-3}$ \\
$k_{\mathrm{th}}\left(\mathrm{W} \mathrm{mm}^{-1} \mathrm{~K}^{-1}\right)$ & 0.01 \\
$c\left(\mathrm{Jg} \mathrm{K}^{-1}\right)$ & 1500 \\
Young modulus $(\mathrm{MPa})$ & 3000 \\
Poisson coefficient & 0.25 \\
$C_{\mathrm{n}}(\mathrm{MPa})$ & $10^{5}$ \\
$C_{t}(\mathrm{MPa})$ & $10^{5}$ \\
$w\left(\mathrm{~J} / \mathrm{mm}^{-1}\right)$ & 0.1 \\
$b\left(\mathrm{~N} \mathrm{~ms} \mathrm{~mm} \mathrm{~mm}^{-1}\right)$ & 0 \\
\hline
\end{tabular}

Simulations are performed for different values of the cohesion $\gamma\left(\gamma \in\left\{0 ; 3.8 \times 10^{-5} ; 3.8 \times 10^{-4} ; 3.8 \times 10^{-3}\right.\right.$; $\left.\left.3.8 \times 10^{-2} ; 3.8 \times 10^{-1}\right\}\right)$, and different values of the couple $(P, V)$ are used $((P, V) \in\{(1,10) ;(2,5) ;(5,2)$; $(10,1)\})$, keeping the product $P V$ constant.

\subsection{Numerical Measures}

As interactions between particles are based on CZM, the status of an interaction is broken or not broken. As the $\beta_{\alpha}$ variable, related to contact $\alpha$, represents the transition between the two states, it is interesting to extend this local variable to more macroscopic quantities. Thus, as proposed in [19], two global damage parameters can be defined.

The first one, denoted $\beta_{i}$, is related to each particle and allows us to characterize the interaction of a particle with its neighborhood. It is defined as:

$\beta_{i}=\frac{1}{n_{i}^{0}} \sum_{\alpha \in \mathcal{L}_{i}} \beta_{\alpha}$,

where $n_{i}^{0}$ and $\mathcal{L}_{i}$ are, respectively, the initial number of contacts and the list of contacts of particle $i$. When $\beta_{i}=1$, all first-body interactions are preserved while when $\beta_{i}=0$, all first-body interaction are broken and the particle evolves freely (third-body particles).

The second one is the global damage parameter $\beta$ which is a measure of the integrity of the medium. It is defined as:

$\beta=\frac{1}{n^{0}} \sum_{\alpha=1}^{n^{0}} \beta_{\alpha}$,

where $n^{0}$ is the initial number of contacts in the sample. The more $\beta$ tends to 0 , the more the sample is damaged. 
(a)

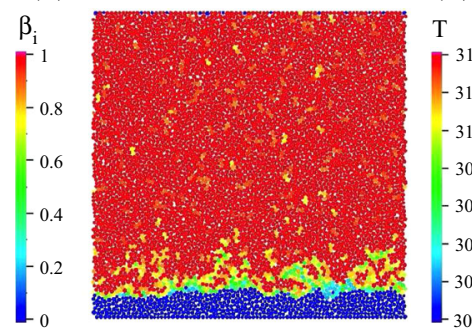

(b)

Fig. 6 Visualization of the damage level (a) and temperature field (b) at the end of simulation ( $10 \mathrm{~ms}$ ) for $P=10 \mathrm{MPa}, V=1 \mathrm{~m} / \mathrm{s}$ and $\gamma=0.038 \mathrm{~N}$

In addition to the damage parameter, the global friction coefficient $\mu$ is defined as:

$\mu(t)=\frac{F_{t}(t)}{F_{n}}$,

which represents the ratio between the force in the sliding direction (resulting from the sliding motion) and the normal force applied on the upper wall.

Figure 6 presents a typical snapshot of the sample at the end of simulation.

The damaging parameter $\beta_{i}$ (cf Fig. 6a) reveals the creation of a third-body layer which allows adaption of the relative motions between the bulk and the moving boundary. The temperature field (cf Fig. 6b) shows a strong localization of heat within the third body and a large variation between thermal response of the first and the third body.

Thus according to the tribological circuit, the damage of the first body represent the source flow, while the thirdbody evolution represent the internal flow. As periodic conditions lead to keep all particles within the contact, the wear flow is equal to zero.

\subsection{Effect of Third-Body Cohesion}

First of all, the effect of the third-body cohesion on the mechanical and thermal properties is investigated. To analyze such an effect, the dimensionless parameter $\eta$, defined as $\eta=\gamma / P d$, is preferred to be taken as a measure of the cohesion $\gamma$.

For pure discontinuous media, as for granular systems, the influence of the cohesive force $\gamma$ on the macroscopic friction is well known [26]. A transition on the macroscopic friction has been observed for a value of $\eta$ around 0.1 .

In the present case, for a normal pressure of $10 \mathrm{MPa}$, if $\gamma \in[0,0.38 \mathrm{~N}], \eta$ varies from 0 to 1 . Figure 7 presents evolution of the macroscopic friction coefficient during the simulation process for different values of $\eta$.

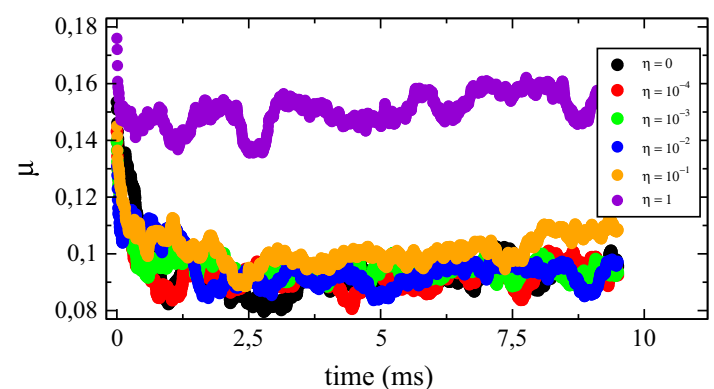

Fig. 7 Evolution of macroscopic friction coefficient during the simulation

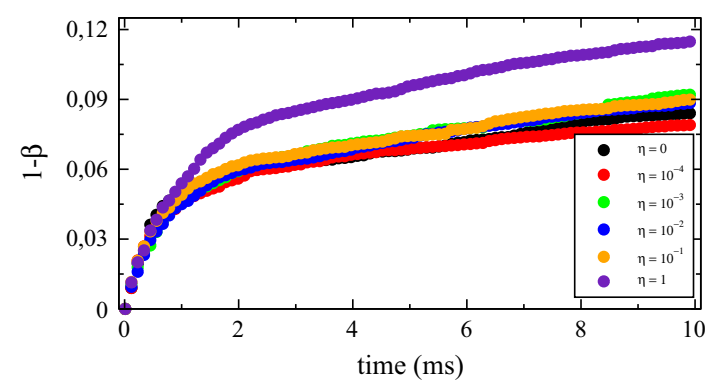

Fig. 8 Evolution of global damage during the simulation

Except for the higher value of $\eta$, evolution is the same for the other values. The friction decreases strongly during the first time of the simulation, then increases slowly. Contrary to earlier results for a fully discrete medium [26], the behavior transition occurs for higher value of $\eta$. Nevertheless, the friction coefficient does not seem to be in a stationary regime. At the end of the simulation, the friction values obtained with a value of $\eta$ equal to 0.1 are larger than the ones obtained with lower values. Simulations should be run further to see if this difference continues.

In parallel to such observations, sample degradation of the global damage evolution $\beta$ is observed (cf Fig. 8).

This evolution is decomposed in two parts, independently of the value of $\eta$. A fast increase in the global damage is observed in the first time of the simulation. Then, approximately around $2 \mathrm{~ms}$, the slope of global damage evolution changes completely (to be less stiff). This change of slope is due to the creation of the third-body layer located between the bulk and the moving boundary which accommodates the velocity. This accommodation preserves the bulk from severe degradation but also minimizes the energy dissipation (decrease in friction coefficient). Note that, as for friction coefficient, evolution of the global damage for a value of $\eta$ equals to one is different than for other values of $\eta$. A large cohesion intensity value has a strong impact at the beginning of the simulation (transient regime). Once the third-body layer is created, 


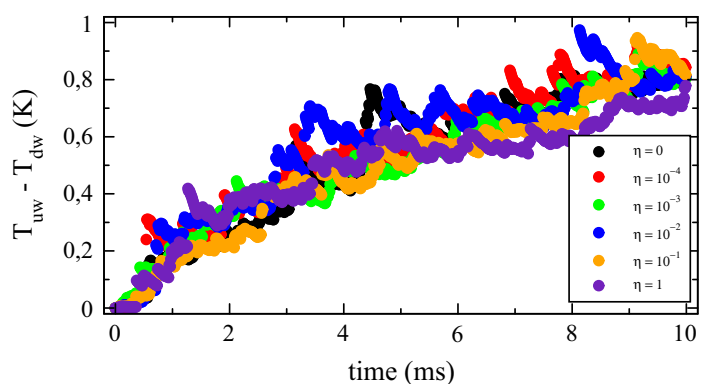

Fig. 9 Evolution of temperature difference between the upper $T_{\mathrm{uw}}$ and lower $T_{\mathrm{lw}}$ boundaries during the simulation

this impact is not so clear. Note, that at the end of the simulation, no steady state has been yet reached for any value of $\eta$. Longer simulations should be performed to obtain steady-state behavior.

In complement with mechanical observation, thermal measures are performed. The first observed quantity is the temperature difference between the upper and lower boundaries (cf Fig. 9).

As expected, this difference increases with time, but it appears that there is no significant influence of $\eta$ on such evolution. According to this global measure, the third-body rheology does not affect the difference of temperature between boundaries.

To complete this global observation, the maximal value of the temperature deviation, denoted $\Delta T_{\max }$, is plotted as a function of the damage level $1-\beta$ (cf Fig. 10).

The first general observation is that the more the damage is important (increase in $1-\beta$ ), the more the maximal temperature deviation is important too. As the third-body layer becomes thicker, the number of contacts within the interface becomes larger, leading to an increase in heat generation. But contrary to the boundary thermal difference, an influence of $\eta$ is observed. Particularly, for the highest value of $\eta$ (i.e., $\eta=1$ ), even if the damage level is the most important, the maximal value of the temperature deviation is the lower one. In regard to the friction coefficient evolution, one might expect that the higher is the macroscopic friction, the higher is the maximal temperature deviation. In fact, there is no direct relation between $\mu$ and $\Delta T_{\max }$ (cf Fig. 11), and evolution of the interface should be taken into account.

\subsection{Global Versus Local Variations}

The previous section focuses on the effects of third-body properties (through the parameters $\gamma$ and $d_{w}$ ) on thermal and mechanical macroscopic responses. In this section, the previous properties are determined as function of changes in global solicitations. For such comparisons, different couples $(P, V)$ are used $((P, V) \in\{(1,10) ;(2,5) ;(5,2)$; $(10,1)\})$, keeping the product $P V$ constant and $\gamma$ stays in the same range of values (i.e., $\gamma \in[0,0.38 \mathrm{~N}]$ ).

Figure 12 presents the evolution of the macroscopic friction coefficient as a function of the cohesion intensity $\eta$ for different couples of $(P, V)$ values. The value of $\mu$ is measured in the steady-state regime.

As underlined in the previous section, the macroscopic friction $\mu$ increases with the increase in $\eta$, particulary at higher values of $\eta$. For most sets, the transition in evolution of $\mu$ occurs for values of $\eta$ larger than $10^{-1}$. Moreover, Fig. 12 underlines the combination of two effects related to the simultaneous variations of pressure and velocity. For the same value of $\gamma$, the decrease in $P$ results in an increase in friction for a given value of $\eta$. In addition, the increase in $\mu$, may be related to the increase in $V$ as well as to the decrease in $P$. This kind of observation has been already done by da Cruz et al. [35] for pure discrete media. In their work, evolution of the macroscopic friction is represented as a function of the inertial number $I$ defined as:

$I=\frac{V}{H_{3 c}} \sqrt{\frac{\rho d^{2}}{P}}$,

where $H_{3 c}$ is the thickness of the third-body layer. In all running simulations (cf Fig. 13), after $2 \mathrm{~ms}, H_{3 c}$ is approximately around $4 \%$ of the height of the sample, reaching $6 \%$ at the end of simulation $(t=10 \mathrm{~ms})$.

Thus, after $2 \mathrm{~ms}$ and according to the different values of couple $(P, V)$, the inertial number $I$ ranges between 0.001 and 0.1 . Such a variation of $I$ involves a transition between quasi-static and dynamic regimes, transition involving an increase in $\mu$.

In complement of Fig. 13, the evolution of the thirdbody height at the end of simulation $(t=10 \mathrm{~ms})$ is plotted as a function of the cohesion intensity $\eta$ for the different couples $(P, V)$ (cf Fig. 14).

The third-body thickness appears as constant for values of $\eta$ smaller than $10^{-1}$. This constant value is similar for all couples $(P, V)(<6 \%)$, except for the couple $(P, V)=$ $(10,1)$ which presents a higher constant value. One remarks that for all sets this content value increases for $\eta$ larger than $10^{-1}$. It seems important to underline that the larger third-body thickness does not necessarily lead to the larger coefficient value.

Figure 15 presents the evolution of global damage as a function of cohesion intensity $\eta$ for the different couples $(P, V)$. As it is not possible to have a steady-state regime for such a quantity, only its final value is plotted.

As for friction coefficient, for a given couple $(P, V)$, the global damage $1-\beta$ increases with the increase in $\eta$ and the transition occurs for values of $\eta$ larger than $10^{-1}$. For smaller values of $\eta$, the damage values are approximately 


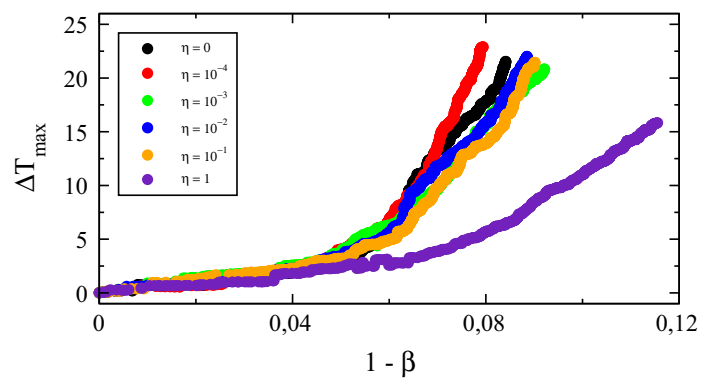

Fig. 10 Maximal temperature deviation as a function of the global damage

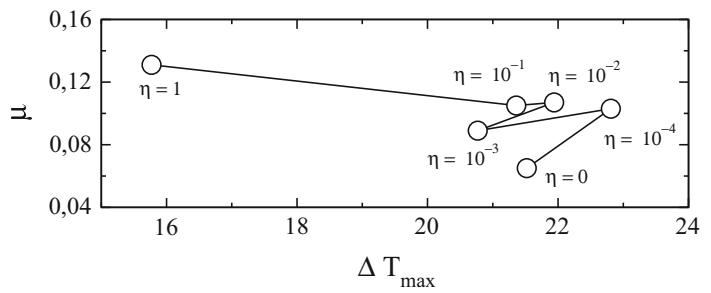

Fig. 11 Maximal temperature deviation versus macroscopic friction for the different values of $\eta$ at the end of the simulation $(t=10 \mathrm{~ms})$

equal. But contrary to the friction, the damage increases when $P$ increases and $V$ decreases. Only the values of damage obtained with the higher value of $P$ are apart and higher than the other. The large pressure creates the most important third-body layer which becomes larger when $\eta$ increases. Such a layer is large enough to accommodate the shearing process and consequently minimize the friction (cf Fig. 12).

To complement the mechanical observations, Fig. 16 presents evolution of the maximal value of temperature deviation as a function of the cohesion intensity $\eta$ for the different couples $(P, V)$. As for damage (cf Fig. 15), the value measured at the end of the simulation is plotted.

Observations are similar to those for friction: an increase in $\Delta T_{\max }$ related to the increase in $V$ but also to the decrease in $P$. Contrary to mechanical observations, an increase in $\eta$ does not necessarily increase the maximal value of temperature. For the couple $(P, V)$ equal to $(10,1)$ and $(5,2), \Delta T_{\max }$ decreases when $\eta$ increases. The reverse behavior occurs for the couple $(P, V)$ equal to $(2,5)$ and $(1,10)$. It is interesting to note that this inversion occurs for a value of the ratio $P / V^{2}$ equal to 1 . If the role of $P$ and $V$ on the mechanical responses is amplified by the cohesion of the third-body layer, independently of their values, it does not have the same behavior for thermal responses. There is competition between the energy due to shearing process and the one due to normal pressure.

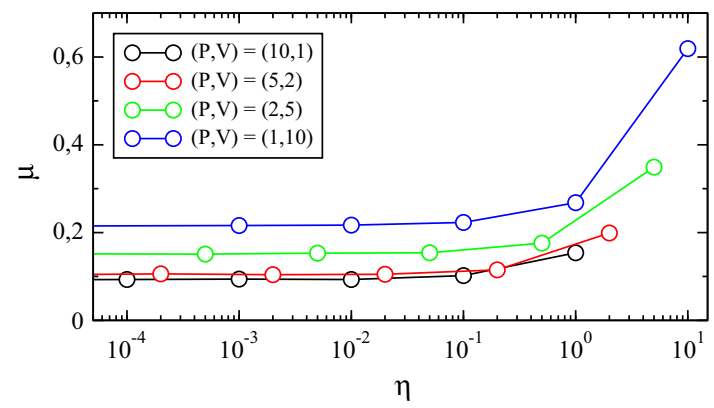

Fig. 12 Evolution of the macroscopic friction coefficient as a function of the cohesion intensity $\eta$ for the different couples $(P, V)$

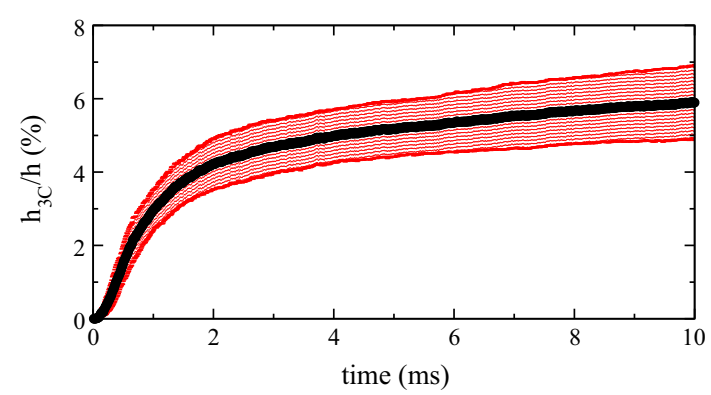

Fig. 13 Average evolution of the third body height for all running simulations; the red area represent the standard deviation domain (Color figure online)

\section{Discussion}

The present paper aims at studying thermo-mechanical phenomena in a tribological interface. An original extended DEM allows the transition between first and third-bodies from a thermo-mechanical point of view. For static continuous heated samples, a good correlation is observed with a similar static example run with finite-element method.

Besides, tribological conditions are applied on a homogeneous sample where damage of first body is governed by CZM and third-body debris is controlled by cohesive laws. First-body CZMs are based on two parameters (the stiffness $C_{\mathrm{n}}$ and the decohesion energy $w$ ); CZM friction and viscosity are secondary as the packing density is already guaranteed by both the high volume fraction of the packing and the high normal stiffness. Third-body local friction is also neglected as its influence on the macroscopic friction is secondary in regard to cohesion and roughness used in the model [26].

During solicitations where cohesive force, pressure or velocity varies, the first body is damaged more or less quickly to create a third-body layer. Their relative proportions and kinematics modulate heat generation and transfer. The local convective and radiative heat flux are neglected as they are not of the first order [16]. 


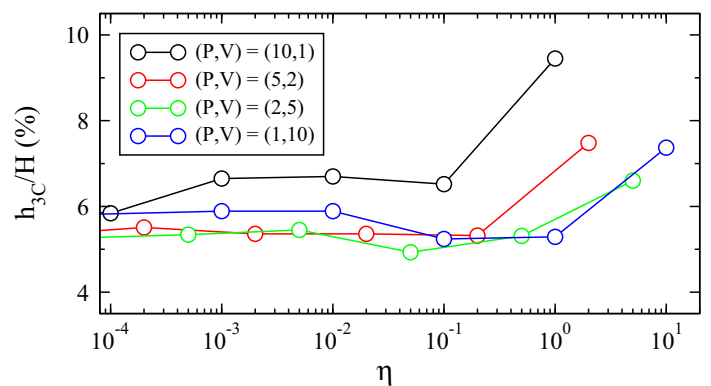

Fig. 14 Evolution of the third-body height at the end of simulation $(t=10 \mathrm{~ms})$ as a function of the cohesion intensity $\eta$ for the different couples $(P, V)$

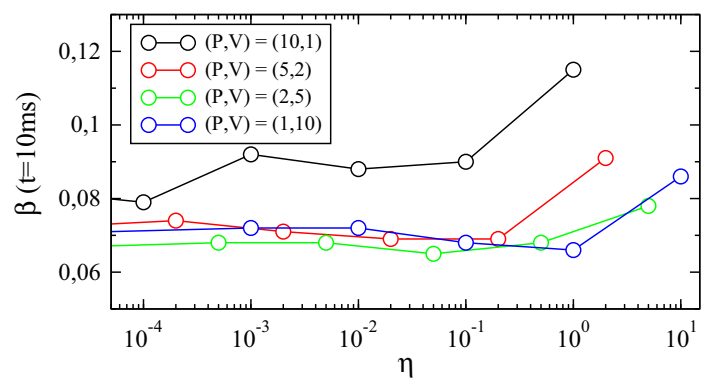

Fig. 15 Evolution of the global damage at the end of simulation $(t=10 \mathrm{~ms})$ as a function of the cohesion intensity $\eta$ for the different couples $(P, V)$

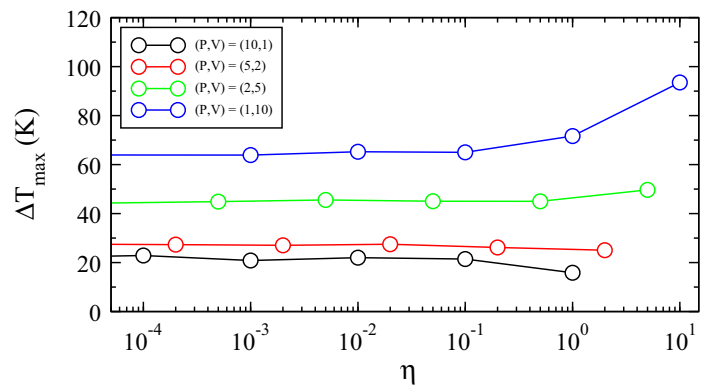

Fig. 16 Evolution of the maximal value of temperature deviation as a function of the cohesion intensity $\eta$ for the different couples $(P, V)$

Through simulations, let us recapitulate the main facts about variation of cohesive force or pressure and velocity.

Cohesive force is an insightful parameter which represents a physicochemical aspect occurring for third-body particles. It allows us to avoid the use of arbitrary local friction coefficient between third-body particles. It shows a threshold effect on damaging, rheological and thermal behaviors. From a certain value of cohesive force, damage is more intense and friction coefficient notably increases. It causes a decrease in maximal temperature despite more interactions. That point comes from a thick third body which facilitates heat conduction between particles of third body. Consequently, choice of cohesive force value is crucial and heat conduction and transfer are in balance to master heat in a contact.

Even if the applied energy to the system is constant but pressure and velocity evolves, impacts on tribological behaviors are different. Pressure more controls the damage of first body. Velocity has major role for friction effects because it increases numbers and intensities of collisions between particles of third body. Thus, velocity increases heat generation and, consequently, temperature in a contact. Let us remind that cautions are necessary regarding assumptions of model (periodical conditions) and conclusions would be different if wear is taken into account.

Such numerical simulations allow to identify influence of several parameters (pressure, velocity, cohesive force,...) which take part into a tribological triplet. Considering the evolution of the first and third-bodies involves to distinguish the local energy, including cohesive energy and CZM energy, and the global energy related to the applied pressure and velocity. The contact behavior (according to the tribological triplet definition) relies on a competition between these two quantities. From local versus global energies, heat generation is distinguishable and competes with heat transfer to assess stored or dissipated energies. Work is under progress to understand that energetical point which is relevant to understand the multiphysical complexity occurring in a contact.

Acknowledgments Discrete element simulation have been carried out through open source software platform $L M G C 90$ (https://subver. lmgc.univ-montp2.fr/trac_LMGC90v2/).

\section{References}

1. Kennedy, F.E.: Frictional heating and contact temperatures. In: Brushan, E. (ed.) Modern Tribology Handbook, pp. 1-38. CRC Press, Columbia (2001)

2. Komanduri, R., Hou, H.B.: A review of the experimental techniques for the measurement of heat and temperatures generated in some manufacturing processes and tribology. Tribol. Int. 34, 653-682 (2001)

3. Uetz, H., Fohl, J.: Wear as an energy transformation process. Wear 49, 253-264 (1978)

4. Kennedy, F.E.: Single pass rub phenomena-analysis and experiment. J. Lubr. Technol. 104, 582-588 (1982)

5. Majcherczak, D., Dufrenoy, P., Berthier, Y.: Tribological, thermal and mechanical coupling aspects of the dry sliding contact. Tribol. Int. 40, 834-843 (2007)

6. Barber, J.R.: Thermoelastic instabilities in the sliding of comforming solids. Proc. R. Soc. Lond. A Math. 312, 381-394 (1969)

7. Furey, M.J., Vick, B., Ghasemi, H.M., Bøhn, J.H.: Effects on surface temperatures. Tribol. Int. 40, 595-600 (2007)

8. Kennedy, F.E.: Surface temperature in sliding systems-a finite element analysis. J. Lubr. Technol. 103, 90-96 (1981)

9. Yevtushenko, A.A., Grzes, P.: Finite element analysis of heat partition in a pad/disc brake system. Numer. Heat Transf. A Appl. 59, 521-542 (2011)

10. Olesiak, Z., Pyryev, Y., Yevtushenko, A.: Determination of temperature and wear during braking. Wear 210, 120-126 (1997) 
11. Godet, M.: The third-body approach: a mechanical view of wear. Wear 100, 437-452 (1984)

12. Dayson, C.: Surface temperatures at unlubricated sliding contacts. ASLE Trans. 10, 169-174 (1967)

13. Meresse, D., Harmand, S., Siroux, M., Watremez, M., Dubar, L.: Experimental disc heat flux identification on a reduced scale braking system using the inverse heat conduction method. Appl. Ther. Eng. 48, 202-210 (2012)

14. Ryhming, I.: On temperature and heat source distributions in sliding contact problems. Acta Mech. 32, 261-274 (1979)

15. Jean, M.: The non-smooth contact dynamics method. Comput. Methods Appl. Mech. Eng. 177, 235-257 (1999)

16. Richard, D., Iordanoff, I., Renouf, M., Berthier, Y.: Thermal study of the dry sliding contact with third-body presence. ASME. J. Tribol. 130, 031404 (2008)

17. Berthier, Y.: Background on friction and wear. In: Lemaitre, J. (ed.) Handbook of Materials Behaviour models, pp. 676-699. Elsevier, Amsterdam (2001)

18. Berthier, Y., Vincent, L., Godet, M.: Velocity accommodation in fretting. Wear 125, 25-38 (1988)

19. Champagne, M., Renouf, M., Berthier, Y.: Modeling wear for heterogeneous bi-phasic materials using discrete elements approach. ASME J. Tribol. 136, 021603 (2014)

20. Moreau, J.J.: Unilateral contact and dry friction in finite freedom dynamics. In: Moreau, J.J., Panagiotopoulos, P.D. (eds.) Nonsmooth Mechanics and Applications, pp. 1-82. Springer, Vienna (1988)

21. Cao, H.P., Renouf, M., Dubois, F., Berthier, Y.: Coupling continuous and discontinuous descriptions to model first body deformation in third body flows. ASME J. Tribol. 133, 041601 (2011)

22. Renouf, M., Cao, H.P., Nhu, V.H.: Multiphysical modeling of third-body rheology. Tribol. Int. 44, 417-425 (2011)

23. Renouf, M., Saulot, A., Berthier, Y.: Third-body flow during a wheel-rail interaction. In: Mota Soares, C.A. et al. (eds.) Third
European Conference on Computational Mechanics Solids Proceedings, pp. 5-9. Lisbon, Portugal (2006)

24. Raous, M., Cangémi, L., Cocu, M.: A consistent model coupling adhesion, friction, and unilateral contact. Comput. Methods Appl. Mech. Eng. 177, 383-399 (1999)

25. Frémond, M.: Equilibre des structures qui adhèrent à leur support. C. R. Acad. Sci. Paris 295, 913-916 (1982)

26. Rognon, P., Roux, J.N., Naaim, M., Chevoir, F.: Dense flows of cohesive granular materials. J. Fluid Mech. 596, 21-47 (2008)

27. Vargas, W.L., McCarthy, J.J.: Heat conduction in granular materials. AIChE J. 47, 1052-1059 (2001)

28. Renouf, M., Berthier, Y.: Numerical modeling of heat production and transmission. In: Radjai, F., Dubois, F. (eds.) Discrete-Element Modeling of Granular Materials, pp. 347-366. Wiley-ISTE, London (2011)

29. Cooper, M.G., Mikic, B.B., Yovanovich, M.M.: Thermal contact conductance. Int. J. Heat Mass Transf. 12, 279-300 (1969)

30. Sridhar, M., Yovanovich, M.M.: Thermal contact conductance of tool steel and comparison with model. Int. J. Heat Mass Transf. 39, 831-839 (1996)

31. Laraqi, N., Bairi, A.: Theory of thermal resistance between solids with randomly sized and located contacts. Int. J. Heat Mass Tranf. 45, 4175-4180 (2002)

32. Yovanovitch, M.M.: Thermal contact resistance across elastically deformed spheres. J. Spacecr. Rockets 4, 119-122 (1967)

33. Terreros, I., Iordanoff, I., Charles, J.L.: Simulation of continuum heat conduction using dem domains. Comput. Mater. Sci. 69, 46-52 (2013)

34. Iordanoff, I., Fillot, N., Berthier, Y.: Numerical study of a thin layer of cohesive particles under plane shearing. Powder Technol. 159, 46-54 (2005)

35. da Cruz, F., Emam, S., Prochnowk, M., Roux, J.N., Chevoir, F.: Rheophysics of dense granular materials: discrete simulation of plane shear flows. Phys. Rev. E 72, 021309 (2005) 